\title{
The Stability of a Special Functional Equation with Zero Solution
}

\author{
Lei Jiang ${ }^{+}$ \\ Department of Mathematics,Chengdu Textile College
}

\begin{abstract}
This paper have mainly studied the zero solution stability problem about the first-order functional equation $x^{\prime}(t)=a(t) x(t)+b(t) x\left(t-m_{1}\right)+c(t) x\left(t-m_{2}\right)$. Unifies the Lyapunov stable knowledge, through structure suitable functional, establishes some appropriate inequalities to the functional system's solution, system's zero solution to stabilize identically with the approach stable satisfied condition are obtained.
\end{abstract}

Keywords: differential equation, stability of zero solution, lyapunov function, asymptotic stability.

\section{Introduction}

The problem of the stability of the equation has been the focus of research and discussion in the field of differential equations[1-6].It has been widely used in the fields of mechanics, automatic control, economics and so on.The stability conclusions about differential equations are also applied and extended to several branches, such as functional differential equations and impulsive differential equations.

In this paper, we will discuss the stability of a special functional equation with zero solutions.

$$
x^{\prime}(t)=a(t) x(t)+b(t) x\left(t-m_{1}\right)+c(t) x\left(t-m_{2}\right), t \geq t_{0}
$$

where $a, b, c \in C\left(\left[t_{0}, \infty\right], R\right)$ and $m_{1}>0, m_{2}>0$.

\section{Preliminary Lemma[7-10]}

$$
x^{\prime}(t)=f(t, x)
$$

$x=\operatorname{col}\left(x_{1}, x_{2} \ldots x_{n}\right), f=\operatorname{col}\left(f_{1}, f_{2} \ldots f_{n}\right) \in C\left[G_{H}, R^{n}\right], G_{H}$ guarantees the uniqueness of the solution of the equation (2) and $f(t, 0)=0$.

Lemma 1If there is a positive definite function $V(t, x)$ on a certain area $G_{H}$, the zero solution of the equation (2) is stable.

Lemma 2 When there is the function $V(t, x)$ of the positive definite infinitesimal upper bound in the region $G_{H}=\left\{(t, x) \mid t \geq t_{0},\|x\| \leq H\right\}$, so that $\left.D^{+} V(t)\right|_{(2-1)} \leq 0$ is established, the zero solution of the equation (2) is uniformly stable.

Lemma 3 If there is a positive definite function $V(t, x) \in\left[G_{H}, R^{+}\right]$of infinitesimal upper bound on the domain $G_{H}$, such that $\left.V^{\prime}(t)\right|_{(2-1)}$ is negative definite, the zero solution of the equation (2) is uniformly asymptotically stable.

Lemma 4 In the interval $\left\{t \geq t_{0},\|x\| \leq H\right\}$,

(1) There is a positive definite function $W(x)$, which makes $W^{\prime}(t)$ have upper or lower bounds.

(2) There is a positive definite function $V(t, x) \in C\left[G_{H}, R^{1}\right]$, which makes $\left.V^{\prime}(t)\right|_{(2-1)}$ is negative definite.

\footnotetext{
* Corresponding author. Tel.: + 8613882025709; fax: +86-28-87840049.

E-mail address: roraldo9@163.com.
} 
the zero solution of the equation (2) is asymptotically stable.

Lemma 5 (Hölder's inequality) When $p>1$ and $\frac{1}{p}+\frac{1}{q}=1, \sum_{i=1}^{n}\left|x_{i}\right|\left|y_{i}\right| \leq\left(\sum_{i=1}^{n}\left|x_{i}\right|^{p}\right)^{\frac{1}{p}}\left(\sum_{i=1}^{n}\left|y_{i}\right|^{q}\right)^{\frac{1}{q}}$.

\section{The Zero Solution Stability of the Functional System}

According to the equation $x^{\prime}(t)=a(t) x(t)+b(t) x\left(t-m_{1}\right)+c(t) x\left(t-m_{2}\right), t \geq t_{0}$, we can get the following theorems:

Theorem 1. When $x(t)=x\left(t, t_{0}, \omega\right)$ is a solution of the equation (1) over the point $\left(t_{0}, \omega\right)$, and $x\left(t, t_{0}, \omega\right) \in\left[t_{0}-m, \infty\right), x(s)=\omega(s)\left(s \in[-m, 0], \omega \in C, m=\max \left\{m_{1}, m_{2}\right\}\right.$. Assume $\mu(t)=\exp \left(\int_{t_{0}}^{t} a(s) d s\right)$, If there is a constant $\alpha>0$ that makes $|b(t)| \leq \alpha \mu(t)$ and $|c(t)| \leq \alpha \mu(t), t \geq t_{0}$ are satisfied, there must be.: $|x(t)| \leq V\left(t_{0}\right) \exp \left(\int_{t_{0}}^{t}[a(s)+2 \alpha \mu(s)] d s\right), t \geq t_{0}$, where $V\left(t_{0}\right)=|\omega(0)|+\alpha\left[\int_{-m_{1}}^{0}|\varpi(s)| d s+\int_{-m_{2}}^{0}|\varpi(s)| d s\right]$.

Proof: Due to $\mu(t)=\exp \left(\int_{t_{0}}^{t} a(s) d s\right)$, so, $\mu(t)>0$ and we can get: $\mu^{\prime}(t)=a(t) \mu(t) \leq a(t) \mu(t)+2 \alpha \mu^{2}(t)$.

We define the function: $V(t)=x(t)+\alpha \mu(t)\left[\int_{t-m_{1}}^{t}|x(s)| d s+\int_{t-m_{2}}^{t}|x(s)| d s\right]$.

By calculating the derivative of the solution of $V(t)$ along the equation (1), we can obtain:

$V^{\prime}(t) \leq[a(t)+2 \alpha \mu(t)]|x(t)|+\alpha\left[\int_{t-m_{1}}^{t}|x(s)| d s+\int_{t-m_{2}}^{t}|x(s)| d s\right]\left[a(t) \mu(t)+2 \alpha \mu^{2}(t)\right]$ $=[a(t)+2 \alpha \mu(t)] V(t) \quad\left(V(t)=x(t)+\alpha \mu(t)\left[\int_{t-m_{1}}^{t}|x(s)| d s+\int_{t-m_{2}}^{t}|x(s)| d s\right]\right)$

so, $V(t) \leq V\left(t_{0}\right) \exp \left(\int_{t_{0}}^{t}[a(s)+2 \alpha \mu(s)] d s\right)$, we can get, $|x(t)| \leq V(t) \leq V\left(t_{0}\right) \exp \left(\int_{t_{0}}^{t}[a(s)+2 \alpha \mu(s)] d s\right)$.

Theorem 2 When $x(t)=x\left(t, t_{0}, \omega\right)$ is a solution of the equation (1) over the point $\left(t_{0}, \omega\right)$, and $x\left(t, t_{0}, \omega\right) \in\left[t_{0}-m, \infty\right), x(s)=\omega(s)\left(s \in[-m, 0], \omega \in C, m=\max \left\{m_{1}, m_{2}\right\}\right.$. Assume $\mu(t)=\exp \left(\int_{t_{0}}^{t}[2 a(s)+|b(s)|+|c(s)|] d s\right)$, if there is a constant $\alpha>0$ that makes $|b(t)| \leq \alpha \mu(t)$ and $|c(t)| \leq \alpha \mu(t), t \geq t_{0}$ are satisfied, there must be.: $|x(t)| \leq \sqrt{V\left(t_{0}\right)} \exp \left(\int_{t_{0}}^{t}[a(s)+2 \alpha \mu(s)] d s\right), t \geq t_{0}$ where $V\left(t_{0}\right)=\omega^{2}(0)+\alpha\left[\int_{-m_{1}}^{0} \omega^{2}(0) d s+\int_{-m_{2}}^{0} \omega^{2}(0) d s\right]$.

Proof: Because $\mu(t)=\exp \left(\int_{t_{0}}^{t}[2 a(s)+|b(s)|+|c(s)|] d s\right)$ and $\mu(t)>0$,so,

$$
\mu^{\prime}(t)=[2 a(t)+|b(t)|+|c(t)|] \mu(t) \leq[2 a(t)+|b(t)|+|c(t)|] \mu(t)+2 \alpha \mu^{2}(t)
$$

We define the function: $V(t)=x^{2}(t)+\alpha \mu(t)\left[\int_{t-m_{1}}^{t} x^{2}(s) d s+\int_{t-m_{2}}^{t} x^{2}(s) d s\right]$.

By calculating the derivative of the solution of $V(t)$ along the equation (1), we can obtain:

$$
\begin{aligned}
& V^{\prime}(t) \leq[2 a(t)+2 \alpha \mu(t)] x^{2}(t)+\alpha\left[\int_{t-m_{1}}^{t} x^{2}(s) d s+\int_{t-m_{2}}^{t} x^{2}(s) d s\right]\left[2 a(t) \mu(t)+2 \alpha \mu^{2}(t)\right] \\
& \leq[2 a(t)+|b(t)|+|c(t)|+2 \alpha \mu(t)] V(t)
\end{aligned}
$$


At the same time, we can get the definite integral from $t_{0}$ to $t$,

$$
V(t) \leq V\left(t_{0}\right) \exp \left(\int_{t_{0}}^{t}[2 a(s)+|b(s)|+|c(s)|+2 \alpha \mu(s)] d s\right) \leq V\left(t_{0}\right) \exp \left(\int_{t_{0}}^{t}[2 a(s)+4 \alpha \mu(s)] d s\right)
$$

Owing to $V(t)=x^{2}(t)+\alpha \mu(t)\left[\int_{t-m_{1}}^{t} x^{2}(s) d s+\int_{t-m_{2}}^{t} x^{2}(s) d s\right]$.So, it must be,

$$
|x(t)| \leq \sqrt{V(t)} \leq \sqrt{V\left(t_{0}\right)} \exp \left(\int_{t_{0}}^{t}[a(s)+2 \alpha \mu(s)] d s\right) .
$$

Theorem 3 When $x(t)=x\left(t, t_{0}, \omega\right)$ is a solution of the equation (1) over the point $\left(t_{0}, \omega\right)$, and $x\left(t, t_{0}, \omega\right) \in\left[t_{0}-m, \infty\right), x(s)=\omega(s)\left(s \in[-m, 0], \omega \in C, m=\max \left\{m_{1}, m_{2}\right\}\right.$.

Assume $-\frac{1}{3 r} \leq a(t)+b\left(t+m_{1}\right)+c\left(t+m_{2}\right) \leq-m_{1} b^{2}\left(t+m_{1}\right)-m_{2} c^{2}\left(t+m_{2}\right)$, there must be.:

(i)If $t \in\left[t_{0}, t_{0}+\frac{r}{2}\right],|x(t)| \leq\|\omega\| \exp \left(\int_{t_{0}}^{t} a(s) d s\right)\left[1+\int_{t_{0}}^{t_{0}+\frac{r}{2}}(|b(s)|+|c(s)|)\right]$;

(ii)If $t \geq t_{0}+\frac{r}{2},|x(t)| \leq 3 \sqrt{2 V\left(t_{0}\right)} \exp \left(\frac{1}{2} \int_{t-\frac{r}{2}}^{t}\left[a(s)+b\left(s+m_{1}\right)+c\left(s+m_{2}\right)\right] d s\right)$.

where $V\left(t_{0}\right)=\left[\omega(0)+\int_{t_{0}-m_{1}}^{t_{0}} b\left(s+m_{1}\right) x(s) d s+\int_{t_{0}-m_{2}}^{t_{0}} c\left(s+m_{2}\right) x(s) d s\right]^{2}$

$$
+\int_{-m_{1}}^{0} \int_{t_{0}+s}^{t_{0}} b^{2}\left(u+m_{1}\right) x^{2}(u) d u d s+\int_{-m_{2}}^{0} \int_{t_{0}+s}^{t_{0}} c^{2}\left(u+m_{2}\right) x^{2}(u) d u d s
$$

Proof: $(i)$ If $t \in\left[t_{0}, t_{0}+\frac{r}{2}\right]$, the equation (1) can be re written as:

$x^{\prime}(t)=a(t) x(t)+b(t) \alpha(t)+c(t) \beta(t) \quad\left(\beta(t)=\omega\left(t-t_{0}-m_{2}\right)\right.$

we can get: $x(t)=\exp \left(\int_{t_{0}}^{t} a(s) d s\right)\left\{\omega\left(t_{0}\right)+\int_{t_{0}}^{t}\left(b(u) \alpha(u)+c(u) \beta(u) \exp \left(-\int_{t_{0}}^{t} a(s) d s\right) d u\right\}\right.$

So, when $t \in\left[t_{0}, t_{0}+\frac{r}{2}\right]$, there must be: $|x(t)| \leq\|\omega\| \exp \left(\int_{t_{0}}^{t} a(s) d s\right)\left[1+\int_{t_{0}}^{t_{0}+\frac{r}{2}}(|b(s)|+|c(s)|)\right], t \geq t_{0}$.

(ii) We define the function:

$$
\begin{aligned}
V(t)=\left[x(t)+\int_{t-m_{1}}^{t} b\left(s+m_{1}\right) x(s) d s+\int_{t-m_{2}}^{t} c\left(s+m_{2}\right) x(s) d s\right]^{2} \\
\quad+\int_{-m_{1}}^{0} \int_{t+s}^{t} b^{2}\left(u+m_{1}\right) x^{2}(u) d u d s+\int_{-m_{2}}^{0} \int_{t+s}^{t} c^{2}\left(u+m_{2}\right) x^{2}(u) d u d s
\end{aligned}
$$

By calculating the derivative of the solution of $V(t)$ along the equation (1), we can obtain:

$$
\begin{aligned}
& V^{\prime}(t)=\left[a(t)+b\left(t+m_{1}\right)+c\left(t+m_{2}\right)\right]\left[x^{2}(t)+2 x(t) \int_{t-m_{1}}^{t} b\left(s+m_{1}\right) x(s) d s+2 x(t) \int_{t-m_{2}}^{t} c\left(s+m_{2}\right) x(s) d s\right. \\
& \left.+\left(\int_{t-m_{1}}^{t} b\left(s+m_{1}\right) x(s) d s\right)^{2}+\left(\int_{t-m_{1}}^{t} c\left(s+m_{1}\right) x(s) d s\right)^{2}+2 \int_{t-m_{1}}^{t} b\left(s+m_{1}\right) x(s) \int_{t-m_{2}}^{t} c\left(s+m_{2}\right) x(s) d s\right] \\
& +\left[a(t)+b\left(t+m_{1}\right)+c\left(t+m_{2}\right)+m_{1} b^{2}\left(t+m_{1}\right)+m_{2} c^{2}\left(t+m_{2}\right)\right] x^{2}(t) \\
& -\left[a(t)+b\left(t+m_{1}\right)+c\left(t+m_{2}\right)\right]\left[\int_{t-m_{1}}^{t} b\left(s+m_{1}\right) x(s) d s+\int_{t-m_{1}}^{t} c\left(s+m_{1}\right) x(s) d s\right]^{2} \\
& -\int_{t-m_{1}}^{t} b^{2}\left(s+m_{1}\right) x^{2}(s) d s-\int_{t-m_{2}}^{t} c^{2}\left(s+m_{2}\right) x^{2}(s) d s \\
& \left.=\left[a(t)+b\left(t+m_{1}\right)+c\left(t+m_{2}\right)\right]\right\}\left[x(t)+\int_{t-m_{1}}^{t} b\left(s+m_{1}\right) x(s) d s+\int_{t-m_{2}}^{t} c\left(s+m_{2}\right) x(s) d s\right]^{2} \\
& \left.+\int_{-m_{1}}^{0} \int_{t+s}^{t} b^{2}\left(u+m_{1}\right) x^{2}(u) d u d s+\int_{-m_{2}}^{0} \int_{t+s}^{t} c^{2}\left(u+m_{2}\right) x^{2}(u) d u d s\right\}
\end{aligned}
$$


$+\left[a(t)+b\left(t+m_{1}\right)+c\left(t+m_{2}\right)+m_{1} b^{2}\left(t+m_{1}\right)+m_{2} c^{2}\left(t+m_{2}\right)\right] x^{2}(t)$

$-\left[a(t)+b\left(t+m_{1}\right)+c\left(t+m_{2}\right)\right\}\left\{\left[\int_{t-m_{1}}^{t} b\left(s+m_{1}\right) x(s) d s+\int_{t-m_{2}}^{t} c\left(s+m_{2}\right) x(s) d s\right]^{2}\right.$

$\left.+\int_{-m_{1}}^{0} \int_{t+s}^{t} b^{2}\left(u+m_{1}\right) x^{2}(u) d u d s+\int_{-m_{2}}^{0} \int_{t+s}^{t} c^{2}\left(u+m_{2}\right) x^{2}(u) d u d s\right\}$

$-\int_{t-m_{1}}^{t} b^{2}\left(s+m_{1}\right) x^{2}(s) d s-\int_{t-m_{2}}^{t} c^{2}\left(s+m_{2}\right) x^{2}(s) d s$

Because $a(t)+b\left(t+m_{1}\right)+c\left(t+m_{2}\right) \leq-m_{1} b^{2}\left(t+m_{1}\right)-m_{2} c^{2}\left(t+m_{2}\right)$

So, $a(t)+b\left(t+m_{1}\right)+c\left(t+m_{2}\right)+m_{1} b^{2}\left(t+m_{1}\right)+m_{2} c^{2}\left(t+m_{2}\right) \leq 0$

So we can get the following conclusions:

$V^{\prime}(t) \leq\left[a(t)+b\left(t+m_{1}\right)+c\left(t+m_{2}\right)\right] V(t)$

$-\left[a(t)+b\left(t+m_{1}\right)+c\left(t+m_{2}\right)\right]\left\{\left[\int_{t-m_{1}}^{t} b\left(s+m_{1}\right) x(s) d s+\int_{t-m_{2}}^{t} c\left(s+m_{2}\right) x(s) d s\right]^{2}\right.$

$\left.+\int_{-m_{1}}^{0} \int_{t+s}^{t} b^{2}\left(u+m_{1}\right) x^{2}(u) d u d s+\int_{-m_{2}}^{0} \int_{t+s}^{t} c^{2}\left(u+m_{2}\right) x^{2}(u) d u d s\right\}$

$-\int_{t-m_{1}}^{t} b^{2}\left(s+m_{1}\right) x^{2}(s) d s-\int_{t-m_{2}}^{t} c^{2}\left(s+m_{2}\right) x^{2}(s) d s$

Meanwhile, $\left(\int_{t-m_{1}}^{t} b\left(s+m_{1}\right) x(s) d s+\int_{t-m_{1}}^{t} c\left(s+m_{1}\right) x(s) d s\right)^{2}$

$\leq 2 \max \left\{m_{1}, m_{2}\right\}\left[\int_{t-m_{1}}^{t} b^{2}\left(s+m_{1}\right) x^{2}(s) d s+\int_{t-m_{1}}^{t} c^{2}\left(s+m_{1}\right) x^{2}(s) d s\right]$

$=2 m\left[\int_{t-m_{1}}^{t} b^{2}\left(s+m_{1}\right) x^{2}(s) d s+\int_{t-m_{1}}^{t} c^{2}\left(s+m_{1}\right) x^{2}(s) d s\right] ;$

$\int_{-m_{1}}^{0} \int_{t+s}^{t} b^{2}\left(u+m_{1}\right) x^{2}(u) d u d s \leq m_{1} \int_{t-m_{1}}^{t} b^{2}\left(u+m_{1}\right) x^{2}(u) d u$

$\leq \max \left\{m_{1}, m_{2}\right\} \int_{t-m_{1}}^{t} b^{2}\left(u+m_{1}\right) x^{2}(u) d u=m \int_{t-m_{1}}^{t} b^{2}\left(u+m_{1}\right) x^{2}(u) d u$

$\int_{-m_{2}}^{0} \int_{t+s}^{t} c^{2}\left(u+m_{1}\right) x^{2}(u) d u d s \leq m_{2} \int_{t-m_{2}}^{t} c^{2}\left(u+m_{1}\right) x^{2}(u) d u$

$\leq \max \left\{m_{1}, m_{2}\right\} \int_{t-m_{2}}^{t} c^{2}\left(u+m_{1}\right) x^{2}(u) d u=m \int_{t-m_{2}}^{t} c^{2}\left(u+m_{1}\right) x^{2}(u) d u$

So, $-\int_{t-m_{1}}^{t} b^{2}\left(s+m_{1}\right) x^{2}(s) d s-\int_{t-m_{2}}^{t} c^{2}\left(s+m_{2}\right) x^{2}(s) d s$

$\leq-\frac{1}{3 m}\left[\left(\int_{t-m_{1}}^{t} b\left(s+m_{1}\right) x(s) d s+\int_{t-m_{1}}^{t} c\left(s+m_{1}\right) x(s) d s\right)^{2}\right.$

$\left.+\int_{-m_{1}}^{0} \int_{t+s}^{t} b^{2}\left(u+m_{1}\right) x^{2}(u) d u d s+\int_{-m_{2}}^{0} \int_{t+s}^{t} c^{2}\left(u+m_{2}\right) x^{2}(u) d u d s\right]$

There must be: $\frac{1}{3 m}\left[\left(\int_{t-m_{1}}^{t} b\left(s+m_{1}\right) x(s) d s+\int_{t-m_{1}}^{t} c\left(s+m_{1}\right) x(s) d s\right)^{2}\right.$

$\left.+\int_{-m_{1}}^{0} \int_{t+s}^{t} b^{2}\left(u+m_{1}\right) x^{2}(u) d u d s+\int_{-m_{2}}^{0} \int_{t+s}^{t} c^{2}\left(u+m_{2}\right) x^{2}(u) d u d s\right]$

$-\int_{t-m_{1}}^{t} b^{2}\left(s+m_{1}\right) x^{2}(s) d s-\int_{t-m_{2}}^{t} c^{2}\left(s+m_{2}\right) x^{2}(s) d s \leq 0$

Since $-\frac{1}{3 r} \leq a(t)+b\left(t+m_{1}\right)+c\left(t+m_{2}\right)$, so,

$-\left[a(t)+b\left(t+m_{1}\right)+c\left(t+m_{2}\right)\right]\left\{\left[\int_{t-m_{1}}^{t} b\left(s+m_{1}\right) x(s) d s+\int_{t-m_{2}}^{t} c\left(s+m_{2}\right) x(s) d s\right]^{2}\right.$ 
$\left.+\int_{-m_{1}}^{0} \int_{t+s}^{t} b^{2}\left(u+m_{1}\right) x^{2}(u) d u d s+\int_{-m_{2}}^{0} \int_{t+s}^{t} c^{2}\left(u+m_{2}\right) x^{2}(u) d u d s\right\}$

$-\int_{t-m_{1}}^{t} b^{2}\left(s+m_{1}\right) x^{2}(s) d s-\int_{t-m_{2}}^{t} c^{2}\left(s+m_{2}\right) x^{2}(s) d s$

$\leq \frac{1}{3 m}\left\{\left[\int_{t-m_{1}}^{t} b\left(s+m_{1}\right) x(s) d s+\int_{t-m_{2}}^{t} c\left(s+m_{2}\right) x(s) d s\right]^{2}\right.$

$\left.+\int_{-m_{1}}^{0} \int_{t+s}^{t} b^{2}\left(u+m_{1}\right) x^{2}(u) d u d s+\int_{-m_{2}}^{0} \int_{t+s}^{t} c^{2}\left(u+m_{2}\right) x^{2}(u) d u d s\right\}$

$-\int_{t-m_{1}}^{t} b^{2}\left(s+m_{1}\right) x^{2}(s) d s-\int_{t-m_{2}}^{t} c^{2}\left(s+m_{2}\right) x^{2}(s) d s \leq 0 ;$

We can get: $V^{\prime}(t) \leq\left[a(t)+b\left(t+m_{1}\right)+c\left(t+m_{2}\right)\right] V(t)$.

we can get the definite integral from $t_{0}$ to $t$,

$V(t) \leq V\left(t_{0}\right) \exp \left(\frac{1}{2} \int_{t_{0}}^{t}\left[a(s)+b\left(s+m_{1}\right)+c\left(s+m_{2}\right)\right] d s\right), t \geq t_{0}$.

We assume $h>0, \forall q, 0 \leq q \leq h$, According to the mean value theorem of integral, we can get:

$\int_{t-h}^{t} b^{2}\left(u+m_{1}\right) x^{2}(u)(u-t+h) d u \geq \int_{t-q}^{t} b^{2}\left(u+m_{1}\right) x^{2}(u)(u-t+h) d u$

$=(\xi-t+h) \int_{t-q}^{t} b^{2}\left(u+m_{1}\right) x^{2}(u) d u \geq(h-q) \int_{t-q}^{t} b^{2}\left(u+m_{1}\right) x^{2}(u) d u(t-q \leq \xi \leq t)$

As a special case, if $h-q=\frac{h}{2}$, the upper form can be rewritten as:

$\int_{t-h}^{t} b^{2}\left(u+m_{1}\right) x^{2}(u)(u-t+h) d u \geq \frac{h}{2} \int_{t-\frac{h}{2}}^{t} b^{2}\left(u+m_{1}\right) x^{2}(u) d u$

So when the order of integration changes, it holds:

$\int_{-m_{1}}^{0} \int_{t+s}^{t} b^{2}\left(u+m_{1}\right) x^{2}(u) d u d s=\int_{t-m_{1}}^{t} b^{2}\left(u+m_{1}\right) x^{2}(u)\left(u-t+m_{1}\right) d u$

$\geq \frac{m_{1}}{2} \int_{t-\frac{m_{1}}{2}}^{t} b^{2}\left(u+m_{1}\right) x^{2}(u) d u$.

In the same way, we can get :

$\int_{-m_{2}}^{0} \int_{t+s}^{t} c^{2}\left(u+m_{1}\right) x^{2}(u) d u d s=\int_{t-m_{21}}^{t} c^{2}\left(u+m_{1}\right) x^{2}(u)\left(u-t+m_{2}\right) d u \geq \frac{m_{2}}{2} \int_{t-\frac{m_{2}}{2}}^{t} b^{2}\left(u+m_{2}\right) x^{2}(u) d u$.

From the definition of the function $V(t)$, it is easy to get:

$V(t) \geq \frac{m_{1}}{2} \int_{t-\frac{m_{1}}{2}}^{t} b^{2}\left(u+m_{1}\right) x^{2}(u) d u, V(t) \geq \frac{m_{2}}{2} \int_{t-\frac{m_{2}}{2}}^{t} b^{2}\left(u+m_{2}\right) x^{2}(u) d u$

So, $V\left(t-\frac{m_{1}}{2}\right) \geq \frac{m_{1}}{2} \int_{t-m_{1}}^{t-\frac{m_{1}}{2}} b^{2}\left(u+m_{1}\right) x^{2}(u) d u$ and $V\left(t-\frac{m_{2}}{2}\right) \geq \frac{m_{2}}{2} \int_{t-m_{2}}^{t-\frac{m_{21}}{2}} b^{2}\left(u+m_{2}\right) x^{2}(u) d u$;

According to the Holder inequality, we can obtain:

If $t \geq t_{0}+\frac{m}{2}, V(t)+V\left(t-\frac{m_{1}}{2}\right)+V\left(t-\frac{m_{2}}{2}\right)=\left[x(t)+\int_{t-m_{1}}^{t} b\left(s+m_{1}\right) x(s) d s+\int_{t-m_{2}}^{t} c\left(s+m_{2}\right) x(s) d s\right]^{2}$

$+\int_{-m_{1}}^{0} \int_{t+s}^{t} b^{2}\left(u+m_{1}\right) x^{2}(u) d u d s+\int_{-m_{2}}^{0} \int_{t+s}^{t} c^{2}\left(u+m_{2}\right) x^{2}(u) d u d s+V\left(t-\frac{m_{1}}{2}\right)+V\left(t-\frac{m_{2}}{2}\right)$

$\geq \frac{1}{2}\left[x(t)+\int_{t-m_{1}}^{t} b\left(s+m_{1}\right) x(s) d s+\int_{t-m_{2}}^{t} c\left(s+m_{2}\right) x(s) d s\right]^{2}+\frac{m_{1}}{2} \int_{t-\frac{m_{1}}{2}}^{t} b^{2}\left(u+m_{1}\right) x^{2}(u) d u$

$+\frac{m_{2}}{2} \int_{t-\frac{m_{2}}{2}}^{t} b^{2}\left(u+m_{2}\right) x^{2}(u) d u+\frac{m_{1}}{2} \int_{t-m_{1}}^{t-\frac{m_{1}}{2}} b^{2}\left(u+m_{1}\right) x^{2}(u) d u+\frac{m_{2}}{2} \int_{t-m_{2}}^{t-\frac{m_{21}}{2}} b^{2}\left(u+m_{2}\right) x^{2}(u) d u$ 


$$
\begin{aligned}
& \geq \frac{1}{2}\left\{\left[x(t)+\int_{t-m_{1}}^{t} b\left(s+m_{1}\right) x(s) d s+\int_{t-m_{2}}^{t} c\left(s+m_{2}\right) x(s) d s\right]^{2}\right. \\
& +\frac{1}{2}\left(\int_{t-m_{2}}^{t} c\left(s+m_{2}\right) x(s) d s\right)^{2}+\frac{1}{2}\left(\int_{t-m_{1}}^{t} b\left(s+m_{1}\right) x(s) d s\right)^{2} \\
& +\int_{t-m_{2}}^{t} c\left(s+m_{2}\right) x(s) d s \int_{t-m_{1}}^{t} b\left(s+m_{1}\right) x(s) d s \geq \frac{1}{6} x^{2}(t) .
\end{aligned}
$$

Since $V^{\prime}(t) \leq 0$ and $\frac{1}{6} x^{2}(t) \leq V(t)+V\left(t-\frac{m_{1}}{2}\right)+V\left(t-\frac{m_{2}}{2}\right) \leq 3 V\left(t-\frac{r}{2}\right)$,

So, if $t \geq t_{0}+\frac{m}{2}$, there must be:

$$
|x(t)| \leq 3 \sqrt{2 V\left(t-\frac{m}{2}\right)}=3 \sqrt{2 V\left(t_{0}\right)} \exp \left(\frac{1}{2} \int_{t-\frac{r}{2}}^{t}\left[a(s)+b\left(s+m_{1}\right)+c\left(s+m_{2}\right)\right] d s\right) .
$$

\section{Conclusion}

\subsection{If the coefficient of equation (1) satisfies:}

$-\frac{1}{3 r} \leq a(t)+b\left(t+m_{1}\right)+c\left(t+m_{2}\right) \leq-m_{1} b^{2}\left(t+m_{1}\right)-m_{2} c^{2}\left(t+m_{2}\right)$, where $m=\max \left\{m_{1}, m_{2}\right\}$, the zero solution is uniformly stable.

\subsection{If the coefficient of equation (1) satisfies:}

$\int_{t_{0}}^{\infty} b^{2}\left(s+m_{1}\right) d s=\infty$ or $\int_{t_{0}}^{\infty} c^{2}\left(s+m_{2}\right) d s=\infty$, the zero solution is uniformly asymptotically stable.

\section{Acknowledgements}

This paper is supportedby foundation of Sichuan Educational Committee(17ZB0015) and foundation of Chengdu Textile College(2017fzlk03).

\section{References}

[1] G. Di Blasio, K. Kunisch,E. Sinestrari. Stability for abstract linear functional differential equations.Israel Journal of Mathematics.1985, pp. 231-263

[2] TingXiu, Wang. Weakening the condition $W_{1}(|\phi(0)|) \leq V(t, \phi) \leq W_{2}(\|\phi\|)$ for Uniform asymptotic stability.Nonlinear Analysis, Theory, Methods\& Applications.1994,23(2): 251-264.

[3] TX,Wang, Stability in Abstract Functional Differential Equations. Part II. Applications.Journal of Mathematical Analysis \& Applications. 1994,186 (2) :534-558.

[4] R.Dewri, and N.Chakraborti.Simulating recrystallization through cellular automata and genetic algorithms. Modelling \& Simulation in Materials Science \& Engineering. 2004, 13 (3): 173-183.

[5] Masakazu,Onitsuka.Uniform asymptotic stability for damped linear oscillators with variable parameters.Applied Mathematics and Computation.2011,pp. 1436-1442.

[6] D.V. Bel'skii.Investigation of Stability in the Case of Neutral Linear Approximation.Journal of Mathematical Sciences .2016, 215 (3) :285-295.

[7] T.A, Burton. Stability and periodic solutions of ordinary differentialequations. Siam Review.2005.

[8] J.K.Hale. Theory of Functional Differential Equations.Spring-Verlag.1977, 3 (1-2) :81-88.

[9] E.A, Yunkova. Investigation of the stability of linear-systems of neutral type by the Lyapunov function method.Differential Equations.1988,pp.4-431.

[10]X Liu.Stability analysis of switched positive systems: a switched linear copositive Lyapunov function method. IEEE Transactions on Circuits \& Systems II Express Briefs.2009, 56 (5) :414-418. 